\title{
Editorial
}

\section{Agent-based models for economic policy design: Introduction to the special issue}

\section{Agent-based models for economic policy design}

Research in economics has traditionally been (and to a large degree still is) based on the development and analysis of highly stylized, analytically tractable models. However, thanks to the recent developments in computer technology and numerical methods, large-scale simulation is increasingly becoming a powerful and attractive new approach for understanding the characteristics of economic systems and deriving economic policy recommendations. In particular, by explicitly modeling the decentralized interaction of heterogeneous economic agents in systems such as markets, industries or organizations, agent-based computational economics (ACE) attempts to transcend the numerous oversimplifying assumptions underlying most mainstream analytical models. ${ }^{1}$

Recently published summaries of previous ACE work, most notably a volume of the Handbook of Computational Economics dedicated to ACE (Tesfatsion and Judd, 2006), demonstrate that agent-based modeling has not only been employed with success in many different fields of economics, but also that the majority of the existing work is of descriptive rather than normative nature. The aim of this special issue is to focus on the normative potential of the agent-based approach, in particular on the usefulness of ACE models for the evaluation and design of economic policy measures.

Extensive discussions of the potential merits of the agent-based approach for economic modeling can be found, for example, in Pyka and Fagiolo (2007), Tesfatsion (2006), Axtell (2000) and Kirman (1997). Central themes in these discussions are the ability of ACE models to capture explicitly the relationship between structured interaction of heterogeneous individuals and the emerging patterns at the macroeconomic level, and to incorporate different types of boundedly rational individual behavior. In addition, a simulation approach allows us to study the open-ended dynamics (including the transient phase) of the economic system under consideration rather than restrict our attention to the existence and (local) stability analysis of equilibria or characterizations of limit distributions. Most of this discussion is based on a view of ACE models as means for economic theorizing (i.e. as a tool to gain a better understanding of general economic mechanisms in rather abstract settings). Without doubt ACE models have great potential in that domain. In the domain of economic policy, however, it seems that the ability to evaluate policies and institutional changes in rather specific models of particular economic environments (e.g. particular markets and/or industries, specific auction types, etc.) has additionally motivated researchers to rely on ACE models. ${ }^{2}$ An important aspect in this respect is that political decision makers might be more willing to trust findings based on rather detailed simulation models where they see a lot of the economic structure they are familiar with than in general insights obtained in rather abstract mathematical

\footnotetext{
${ }^{1}$ Readers not familiar with the ACE approach are referred to Axelrod and Tesfatsion (2006) or Epstein and Axtell (1996).

${ }^{2}$ Examples include detailed models of energy markets (Sun and Tesfatsion, 2007), the U.S. coffee market (Midgley et al., 1997) or the pharmaceutical industry (Malerba and Orsenigo, 2002).
} 
models. ${ }^{3}$ The papers in this issue illustrate these different approaches. The topics addressed stem from very specific policy design questions to classic general issues in the policy debate.

In spite of encouraging signs, ACE models are still far from being considered as a standard tool for economic policy analysis. Besides typical inertia of the profession to pick up new methods, a number of critical aspects of the ACE approach might be blamed for that. Important issues in that respect are empirical model validation and robustness checks of the derived results. The large flexibility with respect to the setup of agent-based models and the almost unrestricted number of potential model parameters give many degrees of freedom to the modeler and make it difficult to restrict the ranges of model parameters based on empirical data. This poses serious challenges to the use of ACE models for the evaluation and design of economic policy measures. For example, to what extent is the dynamics of the economic system in the simulation model indeed a good representation of the impact it would have in reality? In recent years, different proposals have been made on how to deal with this problem, and although the issue is far from being solved, the emerging literature in this field is starting to give ACE researchers some systematic guidelines about how to deal with empirical validation issues. We refer to Fagiolo et al. (2007) and Windrum et al. (2007) for an extensive discussion of empirical validation of agent-based models.

\section{The papers in this special issue}

The set of papers contained in this special issue is a selection of work presented in July 2005 at the Workshop 'Agent-Based Models for Economics Policy Design' at the Center for Interdisciplinary Research (ZiF) at Bielefeld University. The aim of the workshop was to take stock about what has been done with ACE models in the area of policy design and to discuss the potentials and challenges of the approach. The collection of papers give a good indication of the scope of policy questions, from quite general to very specific, that were addressed and highlight different approaches to deal with issues of validation and robustness checks.

The first three papers of the issue deal with questions of industrial policy and market design. Malerba et al. extend their previous work on 'history-friendly' modelling of the evolution of the computer and the semiconductor industry, using the developed simulation model to study the effect of different types of policies, among others anti-trust policies, entry-support policies or public procurement, on the evolution of industry concentration and the rate of technological change. The paper highlights one additional merit of agent-based modelling, namely the ability to compare within one framework the effects of rather diverse policy measures that would typically be dealt with in different branches of the literature using different types of models.

Micola et al. consider a stylized model of the value chain in electricity markets, where demand on the wholesale market is driven by market outcomes in the retail market. Prices in both markets are determined using uniform price auctions, where firms update their bidding behavior using reinforcement learning. In accordance with realworld observations in many countries the authors assume that wholesalers and retailers are vertically integrated and analyze how interdependencies of rewards for managers in the different business units influence prices and profits on both markets. By considering multi-tier energy markets with netback pricing, the study looks at the problem of the emergence of vertical market power from an innovative angle.

The emergence of different types of bidding behavior in different market environments is also the main topic of the contribution of Duffy and Unver. They simulate the behavior of bidders in two types of auctions, hard or soft close auctions, that differ with respect to the rule governing when the auction is closed. Similar differences in closing rules are present in real-world internet auctions and empirical data show that late bidding is much more frequent in hard-close auctions. The agent-based model of Duffy and Unver is able to reproduce this stylized fact. Furthermore, it allows insights into the properties of the bidding functions responsible for the resulting payoffs on both sides. The authors stress the implications of insights of this type for market design.

The rest of the papers study effects of fiscal policy measures in different parts of the economy.

Assessing the impact of labor market policies at both the aggregate and individual levels is the main goal of the paper by Neugart. More specifically, he develops a multi-sector agent-based model where firms belonging to different sectors require workers with different skills. In order to catch job opportunities arising in sectors for which they are not

\footnotetext{
${ }^{3}$ Moss (2002) discusses the importance of involving the actual decision makers in the process of the generation of agent-based models for policy evaluation.
} 
qualified, workers make human-capital investments that are subsidized by the government through the imposition of taxes on employed workers. Using his agent-based model, Neugart shows that government-financed training measures increase the outflow rate from unemployment, but reduce the outflow rate for those who do not receive subsidies. Therefore, although at the aggregate level the impact of government training subsidies is positive (the unemployment rate decreases), at the individual level these labor market policy programs might lead to potential job displacement effects (e.g. they may harm workers who do not receive government transfers).

Happe et al. use an agent-based agricultural policy simulator to analyze the effect of a regime switch in the way agricultural subsidies are paid on changes in farm structure, prices and farm profits. Their model provides a detailed representation of the farm structure in a region, allowing the authors to highlight the different effects that similar policy measures have in regions with different farm structures. Given the importance of agricultural policy (in particular in the EU) and the heterogeneity of farm structure in many regions, this type of analysis seems to have a large potential for improving actual policy design.

Mannaro et al. challenge the idea that a Tobin tax is able to stabilize foreign exchange and stock markets, thus reducing speculation. To address this issue, they develop an artificial agent-based financial market populated by behaviourally heterogeneous traders with limited resources and study the effect of levying a transaction tax in two setups, one in which traders act in a single market, and another in which there are two related markets. Their extensive simulation exercises show that Tobin-like taxes actually increase volatility and decrease trading volumes.

Chen and Chie address the classical question of determining the tax revenue maximizing tax rate in the framework of lottery markets. Based on an agent-based model where lottery participation of individuals is governed by simple rules that are updated due to social learning, they address the puzzle of why lottery tax rates vary substantially between different countries and lotteries. They show that simulation results indeed provide some explanation for this empirically observed phenomenon. Furthermore, their paper allows insights about the relationship between properties of the individual decision rules and observable patterns such as the effect of the jackpot on lottery participation.

The paper by Wilhite and Allen analyzes the impact of several anti-crime policies dynamically undertaken in artificial societies composed of heterogeneous interacting agents. In their model, individuals, neighborhoods, and cities repeatedly choose how to devote their resources to crime prevention in order to solve the trade-off between costs imposed on the society by criminals and costs associated with fighting crime. Interestingly, their model is able to reproduce (and originally explain) several real-world patterns concerning the emergence and distribution of crime and the intertemporal behavior of criminals. For example, larger cities are shown to develop higher crime rates because larger populations increase the incentives to free-ride on public goods. Furthermore, despite crime decreases with protection spending, the impact of prison turns out to be ambiguous, as a higher rate of imprisonment may lead to more crime in the long run.

Finally, Carayol et al. employ agent-based simulations to study systematically how properties of networks that emerge due to uncoordinated individual link formation decisions compared to those of efficient networks. Based on their insights policies might be designed with the goal to foster the emergence of efficient networks. Given the strong recent interest in the analysis of the formation and the implications of social networks, this seems to be one more very promising area for fruitful normative application of the ACE approach.

\section{References}

Axelrod, R., Tesfatsion, L., 2006. A guide for newcomers to agent-based modeling in the social sciences. In: Tesfatsion, L., Judd, K. (Eds.), Handbook of Computational Economics. II. Agent-based Computational Economics. North Holland, Amsterdam, pp. 1647-1659.

Axtell, R., 2000. Why Agents? On the varied motivation for agent computing in the social sciences. Working Paper No. 17. Center on Social and Economic Dynamics, The Brookings Institution.

Epstein, J., Axtell, R., 1996. Growing Artificial Societies: Social Science from the Bottom Up. MIT Press, Cambridge.

Fagiolo, G., Birchenhall, C., Windrum, P. (Eds.), 2007. Special Issue on Empirical Validation in Agent-Based Models. Computational Economics 30 (3).

Kirman, A., 1997. The economy as an interactive system. In: Arthur, W.B., Durlauf, S.N., Lane, D. (Eds.), The Economy as an Evolving Complex System. II. Addison-Wesley, Boston, pp. 491-532.

Malerba, F., Orsenigo, L., 2002. Innovation and market structure in the dynamics of the pharmaceutical industry and biotechnology: towards a history-friendly model. Industrial and Corporate Change 11, 667-703.

Midgley, D.F., Marks, R.E., Cooper, L.G., 1997. Breeding competitive strategies. Management Science 43, $257-275$.

Moss, S., 2002. Policy analysis from first principles. Proceedings of the National Academy of Sciences 99, 7267-7274. 
Pyka, A., Fagiolo, G., 2007. Agent-based modelling: a methodology for Neo-Schumpeterian economics. In: Hanusch, H., Pyka, A. (Eds.), The Elgar Companion to Neo-Schumpeterian Economics. Edward Elgar, Cheltenham, pp. 467-487.

Sun, J., Tesfatsion, L., 2007. Dynamic testing of wholesale power market designs: an open-source agent-based framework. Computational Economics 30, 291-327.

Tesfatsion, L., 2006. ACE: a constructive approach to economic theory. In: Tesfatsion, L., Judd, K. (Eds.), Handbook of Computational Economics. II. Agent-based Computational Economics. North Holland, Amsterdam, pp. 832-880.

Tesfatsion, L., Judd, K. (Eds.), 2006. Handbook of Computational Economics. II. Agent-based Computational Economics. North Holland, Amsterdam.

Windrum, P., Fagiolo, G., Moneta, A., 2007. Empirical validation of agent-based models: alternatives and prospects. Journal of Artificial Societies and Social Simulation $10(2), 8$.

Herbert Dawid*

Department of Business Administration and Economics, Institute of Mathematical Economics, Bielefeld University, P.O. Box 100131, 33501 Bielefeld, Germany

Giorgio Fagiolo Laboratory of Economics and Management, Sant'Anna School of Advanced Studies, 56127 Pisa, Italy

* Corresponding author. E-mail addresses: hdawid@ wiwi.uni-bielefeld.de (H. Dawid), giorgio.fagiolo@sssup.it (G. Fagiolo)

4 June 2007

Available online 22 April 2008 